\title{
Radio spectra of a sample of X-ray selected BL Lacs
}

\author{
F. Cavallotti ${ }^{1}$, A. Wolter ${ }^{1}$, J. T. Stocke ${ }^{2}$, and T. Rector ${ }^{3, \star}$ \\ 1 Osservatorio Astronomico di Brera, via Brera 28, 20121, Milano, Italy \\ e-mail: cavallot@brera.mi .astro.it \\ 2 Center for Astrophysics and Space Astronomy, University of Colorado, Boulder, CO 80309-0389, USA \\ 3 National Radio Astronomy Observatory, PO Box O, Socorro, NM 87801, USA \\ Received 15 September 2003 / Accepted 18 February 2004
}

\begin{abstract}
We present simultaneous multifrequency radio observations for a complete subsample of 26 XBLs from the Einstein Extended Medium-Sensitivity Survey, obtained with the Very Large Array (VLA). Spectra are computed using fluxes at 20, 6 and $3.6 \mathrm{~cm}$. Unlike many radio selected samples, the EMSS did not impose any criterion on the radio spectrum to identify BL Lac objects. It is therefore possible to investigate the intrinsic radio spectral slope distribution and to determine the effect produced by this selection criterion. We find that $15 \%$ of the observed objects do not meet the flat-spectrum criterion imposed on some other BL Lac samples. A dataset that includes non-simultaneous data (that are also taken with different VLA configurations) shows an even higher percentage of steep spectrum sources. This effect can be ascribed to a larger fraction of extended flux detected with the more compact VLA configuration. Possible biases introduced by the flat-radio-spectrum criterion in the radio-selected BL Lac samples cannot explain the discrepancies observed in the evolutionary properties of Radio and X-ray selected samples of BL Lacs.
\end{abstract}

Key words. galaxies: BL Lacertae objects: general - radio continuum: galaxies

\section{Introduction}

BL Lac objects are an enigmatic class of active galactic nuclei (AGN) characterized by strong radio, optical and X-ray variability, relatively high optical and radio polarization and featureless optical spectra (e.g. Urry \& Padovani 1995). The properties of the class can be explained in terms of the relativistic beaming scenario wherein the observed emission is dominated by Doppler-boosted non-thermal radiation from a relativistic jet aligned with the line of sight (Blandford \& Rees 1978; Antonucci \& Ulvestad 1985).

Owing to their featureless spectra and lack of UV excess in many BL Lacs, optical search techniques (e.g. by excess colors, emission line strength), used to search for other AGN, failed to find BL Lacs in large number (e.g. Fleming et al. 1993). Since BL Lacs are both radio-loud and X-ray-loud, surveys in these frequency bands discover them with high efficiency (e.g. the "1 Jansky" sample: Stickel et al. 1991; the Einstein Extended Medium-Sensitivity Survey (EMSS) sample: Morris et al. 1991; Rector et al. 2000; the Deep X-ray Radio Blazar Survey (DXRBS) sample: Perlman et al. 1998; Landt et al. 2001; the ROSAT All Sky Survey (RASS) sample: Bade et al. 1998; the "sedentary" survey: Giommi et al. 1999; the Radio

\footnotetext{
Send offprint requests to: A. Wolter, e-mail: anna@brera.mi .astro.it

^ Present address: University of Alaska, 3211 Providence Dr., BMB 212, Anchorage, AK 99508, USA.
}

Emitting X-ray (REX) survey sample: Caccianiga et al. 1999, 2002). This splits BL Lacs into two empirical subclasses, namely radio-selected BL Lacs (RBLs) and X-ray-selected BL Lacs (XBLs). Padovani \& Giommi (1995) proposed to distinguish these two classes on a more physical basis, according to the spectral energy distribution (SED). For ease of description here we will refer to RBL and XBL only.

RBLs and XBLs show different behaviors with respect to properties like cosmological evolution, polarization, variability, core-to-extended radio flux ratio, extended radio morphology, spectral features (e.g. Wolter et al. 1994; Stickel et al. 1991; Morris et al. 1991; Jannuzi et al. 1993, 1994; Perlman \& Stocke 1993 to mention just a few) which have not been satisfactorily explained yet. This led to the suggestion that the observed discrepancies are at least partially caused by selection effects due to different criteria used in radio and X-ray surveys.

In particular, the standard selection technique for RBLs requires a flat-radio-spectrum criterion for which the radio spectral slope must be less than $0.5\left(\alpha_{\mathrm{r}} \leq 0.5 ; S_{v} \propto v^{-\alpha_{\mathrm{r}}}\right)$. This criterion has not been used for classical X-ray selected samples of BL Lacs like the EMSS. If surveys with this criterion select only part of the BL Lac population, the RBL samples could be biased and the differences between RBLs and XBLs like the ones mentioned above could be at least partially explained.

It is already known that a number of objects with steep spectra $\left(\alpha_{\mathrm{r}}>0.5\right)$, classified as radio galaxies in radio surveys like the "1 Jansky" (Owen et al. 1996; Perlman et al. 1996), 
otherwise meet the BL Lacs selection criteria, based on optical properties (equivalent width of emission lines; Ca II break contrast) of Marchã et al. (1996), and have broadband properties (radio luminosity and overall spectral energy distributions) that agree with those of BL Lacs (e.g. Rector et al. 2000).

To test if the flat-radio-spectrum criterion really introduces biases in the BL Lacs selection and to estimate the incompleteness degree of RBLs samples, we studied the radio spectral indices of a complete subsample of XBLs in which the identification criteria were not based on the radio spectral properties. Previous multi-frequency studies had already been performed on XBLs. For instance, Laurent-Muehleisen et al. (1993) collected non-simultaneous data for several objects of this class, that however do not make a complete sample. We prefer to deal with the largest subsample for which we could have both simultaneous and non-simultaneous data.

The sources of this subsample are extracted from the EMSS (Gioia et al. 1990; Stocke et al. 1991), a catalog whose BL Lacs sample has been thoroughly studied for completeness (Rector et al. 1999). A pilot study on the radio spectra of 8 XBLs from the EMSS and the HEAO-1 A-2 all-sky survey (Piccinotti et al. 1982) was performed by Stocke et al. (1985). Three objects showed a spectral slope which exceeds the limit for flat spectrum and two more were marginally steep. Preliminary analysis of the EMSS BL Lacs sample, by using non-simultaneous data at 6 and $20 \mathrm{~cm}$ from the EMSS and the NRAO VLA Sky Survey (NVSS: Condon et al. 1998) respectively, showed that about $30 \%$ of the objects have a spectral slope steeper than 0.5. This is the motivation that led us to investigate the EMSS sources by obtaining simultaneous spectra, which are not affected by variability.

We briefly describe the sample of objects used for this work in Sect. 2; in Sect. 3 we present the data reduction process as well as flux densities and radio spectra for each object; in Sect. 4 we show the results obtained; our conclusions are summarized in Sect. 5. Throughout the paper we used $H_{0}=50 \mathrm{~km} \mathrm{~s}^{-1} \mathrm{Mpc}^{-1}$ and $q_{0}=0$, but no conclusions are dependent upon this choice of cosmology.

\section{The sample}

We restrict the analysis to the EMSS north of $\delta=-20^{\circ}$ in order to ensure $100 \%$ identification rate. The revised EMSS BL Lac sample (Rector et al. 2000) then contains 36 BL Lacs from which we observed a subsample of 22 objects, named hereafter CWSR. The sources were selected imposing $f_{6} \mathrm{~cm} \geq 1 \mathrm{mJy}$ in the EMSS dataset and celestial positions that allowed observations at the Very Large Array (VLA ${ }^{1}$ ) during June 2000. The resulting subsample is therefore not biased with respect to radio spectral index.

The VLA observations where performed on June 13 and June 16 in the $L(\lambda=20 \mathrm{~cm}), C(\lambda=6 \mathrm{~cm})$ and $X(\lambda=3.6 \mathrm{~cm})$ frequency bands. The observations were carried out in the

1 The VLA is a facility of the National Radio Astronomy Observatory. The National Radio Astronomy Observatory (NRAO) is a facility of the National Science Foundation operated under cooperative agreement by Associated Universities, Inc.
Table 1. The CWSR sample.

\begin{tabular}{|c|c|c|}
\hline Name & Obs. date & $z$ \\
\hline MS0122.1+0903 & 16 Jun. 2000 & $0.339^{*}$ \\
\hline MS0158.5+0019 & 16 Jun. 2000 & $0.299^{*}$ \\
\hline MS0205.7+3509 & 16 Jun. 2000 & $>0.351^{+}$ \\
\hline MS0257.9+3429 & 16 Jun. 2000 & $0.245^{*}$ \\
\hline MS0317.0+1834 & Jun. 1984 & $0.190^{\dagger}$ \\
\hline MS0419.3+1943 & 16 Jun. 2000 & $0.512^{*}$ \\
\hline MS0607.9+7108 & 16 Jun. 2000 & $0.267^{*}$ \\
\hline MS0737.9+7441 & 16 Jun. 2000 & $0.315^{*}$ \\
\hline MS0922.9+7459 & 13 Jun. 2000 & $0.638^{*}$ \\
\hline MS0950.9+4929 & 13 Jun. 2000 & $\ldots$ \\
\hline MS1019.0+5139 & 13 Jun. 2000 & $0.141^{*}$ \\
\hline MS1050.7+4946 & 13 Jun. 2000 & $0.140^{*}$ \\
\hline MS1207.9+3945 & Jun. 1984 & $0.616^{\dagger}$ \\
\hline MS1221.8+2452 & 13 Jun. 2000 & $0.218^{*}$ \\
\hline MS1229.2+6430 & 13 Jun. 2000 & $0.164^{*}$ \\
\hline MS1235.4+6315 & Jun. 1984 & $0.297^{\dagger}$ \\
\hline MS1402.3+0416 & Jun. 1984 & $0.344:^{\dagger}$ \\
\hline MS1407.9+5954 & 13 Jun. 2000 & $0.495^{*}$ \\
\hline MS1443.5+6348 & 13 Jun. 2000 & $0.299^{*}$ \\
\hline MS1458.8+2249 & 13 Jun. 2000 & $0.235^{*}$ \\
\hline MS1534.2+0148 & 13 Jun. 2000 & $0.312^{*}$ \\
\hline MS1552.1+2020 & 13 Jun. 2000 & $0.222^{*}$ \\
\hline MS1757.7+7034 & 13 Jun. 2000 & $0.407^{*}$ \\
\hline MS2143.4+0704 & 16 Jun. 2000 & $0.237^{*}$ \\
\hline MS2336.5+0517 & 16 Jun. 2000 & $0.74::^{\dagger}$ \\
\hline MS2347.4+1924 & 16 Jun. 2000 & $0.515^{*}$ \\
\hline
\end{tabular}

$\left(^{*}\right)$ Redshift from Morris et al. (1991).

$\left(^{+}\right)$Redshift from Watson et al. (2004).

${ }^{\dagger}$ ) Redshift from Rector et al. (2000).

C configuration. Thus, the maps have an angular resolution equal to $16^{\prime \prime} 3,5^{\prime \prime} .1$ and $3^{\prime \prime}$ at 20,6 and $3.6 \mathrm{~cm}$ respectively. Each object was observed for $\sim 10 \mathrm{~min}$ per frequency, obtaining a sensitivity of about $0.05 \mathrm{mJy}_{\text {beam }}{ }^{-1}$. Flux densities were bootstrapped from 3C 286 (June 13) and 3C 48 (June 16) (Baars et al. 1977)

If we include in the CWSR sample the 4 EMSS objects published in Stocke et al. (1985), observed simultaneously on June 1984 at all the available wavelengths $(20,18,6,2.0$ and $1.3 \mathrm{~cm}$ ) with the VLA in the C/D hybrid configuration, we have 26 sources in total. These sources are listed in Table 1: the first column indicates the source names, the second one the observation dates and the third one the redshift values.

For the same 26 objects we also have non-simultaneous data at 20 and $6 \mathrm{~cm}$, respectively from NVSS and EMSS archives.

\section{Data analysis}

\subsection{Radio maps}

Radio maps for the 22 CWSR sources observed on June 2000 were produced in $\mathcal{A I P S}$ with a standard procedure that 
Table 2. $F_{\text {tot }}, F_{\text {peak }}$ and rms measured in the $L(20 \mathrm{~cm}), C(6 \mathrm{~cm})$ e $X(3.6 \mathrm{~cm})$ frequency band for the 22 XBLs of CWSR sample observed on June 2000 .

\begin{tabular}{|c|c|c|c|c|c|c|c|c|c|}
\hline \multirow[b]{2}{*}{ Name } & \multicolumn{3}{|c|}{$L$ band } & \multicolumn{3}{|c|}{$C$ band } & \multicolumn{3}{|c|}{$X$ band } \\
\hline & $F_{\text {tot }}^{a}$ & $F_{\text {peak }}^{a}$ & $\operatorname{rms}\left(\times 10^{-2}\right)^{b}$ & $F_{\text {tot }}^{a}$ & $F_{\text {peak }}^{a}$ & $\operatorname{rms}\left(\times 10^{-2}\right)^{b}$ & $F_{\text {tot }}^{a}$ & $F_{\text {peak }}^{a}$ & $\operatorname{rms}\left(\times 10^{-2}\right)^{b}$ \\
\hline MS0122.1+0903 & 1.34 & 1.44 & 6.26 & 1.47 & 1.39 & 3.30 & 1.28 & 1.08 & 2.60 \\
\hline MS0158.5+0019 & 13.26 & 12.46 & 8.67 & 9.70 & 8.87 & 3.00 & 8.16 & 7.36 & 3.00 \\
\hline MS0205.7+3509 & 3.53 & 3.53 & 13.85 & 5.24 & 5.04 & 3.40 & 4.96 & 4.24 & 2.49 \\
\hline MS0257.9+3429 & 8.80 & 8.23 & 10.00 & 10.79 & 9.41 & 3.80 & 11.23 & 9.98 & 3.05 \\
\hline MS0419.3+1943 & 6.10 & 5.66 & 8.44 & 9.05 & 7.91 & 3.18 & 8.07 & 7.16 & 2.57 \\
\hline MS0607.9+7108 & 15.33 & 13.12 & 40.71 & 14.37 & 13.97 & 3.58 & 10.34 & 9.82 & 3.74 \\
\hline MS0737.9+7441 & 25.60 & 24.78 & 9.29 & 22.46 & 21.04 & 3.45 & 20.07 & 19.39 & 3.26 \\
\hline MS0922.9+7459 & 43.37 & 21.88 & 8.57 & 4.17 & 3.59 & 4.00 & 2.89 & 2.66 & 3.30 \\
\hline MS0950.9+4929 & 4.58 & 4.56 & 5.67 & 3.42 & 3.16 & 2.88 & 3.25 & 2.89 & 2.72 \\
\hline MS1019.0+5139 & 2.71 & 2.84 & 8.03 & 2.97 & 2.75 & 3.16 & 2.86 & 2.70 & 2.60 \\
\hline MS1050.7+4946 & 57.13 & 48.84 & 7.34 & 42.71 & 39.59 & 3.46 & 38.19 & 32.44 & 3.31 \\
\hline MS1221.8+2452 & 23.31 & 21.17 & 13.02 & 22.00 & 20.95 & 3.50 & 21.59 & 19.80 & 2.75 \\
\hline MS1229.2+6430 & 54.68 & 51.51 & 11.87 & 46.31 & 43.97 & 4.65 & 40.96 & 38.21 & 3.20 \\
\hline MS1407.9+5954 & 31.51 & 24.54 & 12.67 & 19.55 & 16.80 & 4.44 & 16.77 & 13.67 & 3.02 \\
\hline MS1443.5+6348 & 9.74 & 9.55 & 51.29 & 4.47 & 3.88 & 4.69 & 6.29 & 5.11 & 3.17 \\
\hline MS1458.8+2249 & 48.42 & 47.47 & 8.75 & 87.99 & 85.33 & 5.23 & 94.41 & 91.87 & 3.00 \\
\hline MS1534.2+0148 & 65.12 & 60.16 & 8.10 & 34.07 & 25.29 & 3.29 & 24.01 & 15.96 & 2.57 \\
\hline MS1552.1+2020 & 64.46 & 56.92 & 53.89 & 38.79 & 31.93 & 3.79 & 29.61 & 24.06 & 2.71 \\
\hline MS1757.7+7034 & 11.68 & 11.45 & 15.61 & 11.15 & 10.53 & 3.78 & 9.80 & 9.03 & 3.07 \\
\hline MS2143.4+0704 & 78.59 & 65.10 & 15.48 & 51.87 & 45.27 & 6.31 & 43.57 & 37.21 & 2.29 \\
\hline MS2336.5+0517 & 11.82 & 8.83 & 12.62 & 5.86 & 5.26 & 3.30 & 4.69 & 4.38 & 2.80 \\
\hline MS2347.4+1924 & 4.27 & 4.64 & 9.96 & 3.01 & 2.87 & 2.90 & 2.42 & 2.19 & 2.70 \\
\hline
\end{tabular}

${ }^{a}$ Total and Peak Flux Density in mJy.

${ }^{b}$ Root Mean Square in $\mathrm{mJy} \times$ beam $^{-1}$.

includes: removal of RFI from $(u, v)$ data caused by incidental factors during the observations, calibration of all sources in amplitude and in phase, creation of a "cleaned" map of brightness distribution by using a CLEAN algorithm. Particular care was taken at $20 \mathrm{~cm}$ to look and correct for contaminating sources.

For a more stable phase calibration, a calibrator source was observed before and after each source observation. Whenever possible, self-calibration was also applied.

We adopted a uniform weight function. We did some comparison with maps derived from natural weighting function and no significant differences were observed.

The resulting maps do not show morphological structures that are worth publishing. All sources are fitted by Gaussians (even if sometimes with widths larger than the beam size).

\subsection{Flux densities}

\subsubsection{Simultaneous flux densities}

From the "cleaned" maps we computed flux densities and their associated rms errors by assuming the source signal as a Gaussian. We measured both the peak flux density $\left(F_{\text {peak }}\right)$ and the total flux density $\left(F_{\text {tot }}\right)$. We assume that $F_{\text {peak }}$ represents the emission from the source core and we define the extended flux $F_{\text {ext }}$ as the difference between $F_{\text {tot }}$ and $F_{\text {peak }}$.

In Table $2 F_{\text {tot }}, F_{\text {peak }}$ and their associated rms errors are listed for the CWSR sample. By comparing the peak and total flux densities, we confirm that this sample and the $C$ data we obtained follow the BL Lac trend to be compact: we found that the maximum value of the ratio $F_{\text {tot }} / F_{\text {peak }}$ is 1.98 at $20 \mathrm{~cm}$. In Sect. 4.1 we better analyze this aspect.

The noise for each map was determined from the rms fluctuations in areas free of radio emission. We compared these rms errors (see Table 2) with their ideal values $(0.032,0.028$ and 0.023 mJy in $L, C$ and $X$ band respectively) from the VLA manual (Perley 2000). While in $C$ and $X$ bands the rms errors were found similar to their ideal limits, in $L$ band they were larger than their theoretical values. This can be explained by additional interference at $20 \mathrm{~cm}$, and is not a particularly unexpected result.

For 3 sources (MS0122.1+0903, MS1019.0+5139, MS2347+1924) at $20 \mathrm{~cm}$ we measured a total flux density smaller than the peak flux density (but consistent within the errors) probably because of phase errors not completely removed from radio maps by cleaning processes. Due to the small numerical difference between $F_{\text {tot }}$ and $F_{\text {peak }}\left(F_{\text {tot }} / F_{\text {peak }}\right.$ equal to $0.93,0.95$ and 0.92 respectively) and since BL Lacs are known as objects with a high core-to-extended ratio (e.g. Perlman \& Stocke 1993), this does not introduce significant biases.

In three sources (namely MS0607.9+7108, MS1443.5+6348, MS1552+2020) we measured a very high rms threshold because of a source in the neighborhood, 
Table 3. $F_{\text {tot }}$ and rms reported from Stocke et al. (1985) for the 4 EMSS objects included in the CWSR sample.

\begin{tabular}{c|rrrrr}
\hline \hline & \multicolumn{5}{|c}{$F_{\text {tot }}(\mathrm{mJy})$} \\
Name & $20 \mathrm{~cm}$ & $18 \mathrm{~cm}$ & $6 \mathrm{~cm}$ & $2 \mathrm{~cm}$ & $1.3 \mathrm{~cm}$ \\
\hline MS0317.0+1834 & $17.3 \pm 0.6$ & $16.5 \pm 0.8$ & $11.5 \pm 0.23$ & $5.5 \pm 0.3$ & $<4.5$ \\
MS1207.9+3945 & $15.1 \pm 1.1$ & $18.3 \pm 1.3$ & $6.1 \pm 0.4$ & $0.9 \pm 0.3$ & $<4.2$ \\
MS1235.4+6315 & $10.7 \pm 0.5$ & $10.1 \pm 0.5$ & $7.0 \pm 0.38$ & $4.6 \pm 0.3$ & Not Obs. \\
MS1402.3+0416 & $35.5 \pm 1.3$ & $38.2 \pm 1.4$ & $20.8 \pm 0.4$ & $14.5 \pm 0.3$ & $8.7 \pm 1.4$ \\
\hline
\end{tabular}

either very bright or extended, that could not be properly cleaned. In Table 3 we report simultaneous data for the 4 objects included in the CWSR sample from Stocke et al. (1985). The total flux density $F_{\text {tot }}$ and their associated rms errors are listed at all the observed wavelengths.

\subsubsection{Non-simultaneous flux densities}

Most datasets available to compute radio spectra are not simultaneous; moreover, BL Lacs are known to be variable objects (e.g. Angel \& Stockman 1980; Ulrich et al. 1995). To assess the impact of variability on non-simultaneous measurements of $\alpha_{\mathrm{r}}$, we statistically compare the $\alpha_{\mathrm{r}}$ values measured from simultaneous data to values determined with nonsimultaneous data. We used $20 \mathrm{~cm}$ NVSS (Condon et al. 1998) and $6 \mathrm{~cm}$ EMSS (Maccacaro et al. 1994) catalog fluxes. Since MS0122.1+0903 and MS0419.3+1943 do not appear in the NVSS catalog (they are at the completeness limit) we measured their fluxes from the NVSS on-line radio images which can be down-loaded in FITS format (see http://www.cv.nrao.edu/NVSS/postage.html).

In Table 4 flux densities are shown for the 26 listed objects.

\subsection{Radio spectra}

By using the multi-frequency flux densities measured (Tables 2 and 3) we construct simultaneous spectra for CWSR sources. The spectral shape is assumed to be a power law $\left(S_{v} \propto\right.$ $\left.v^{-\alpha_{\mathrm{r}}}\right)$. The slopes were computed with the Least Square Method (LSQ) and are reported in Table 5. At $20 \mathrm{~cm}$ the MS0922.9+7459 radio map is confused due to the superposition of another source (probably a radio galaxy in a foreground cluster). Thus, the corresponding flux density reported in this work, even if it is the best measure we could obtain, is to be considered as an upper limit and was not included in all the spectral slope calculations for this source.

To investigate whether variability could affect the spectral slope, we calculated simultaneous and non-simultaneous two-point $(20 \mathrm{~cm}+6 \mathrm{~cm}) \alpha_{\mathrm{r}}$ values with the EMSS $(6 \mathrm{~cm})$, NVSS $(20 \mathrm{~cm})$ and June $2000(20 \mathrm{~cm}+6 \mathrm{~cm})$ observations for the CWSR sources.

We computed as well the spectral index for the source cores, finding no significant changes with respect the $\alpha_{\mathrm{r}}$ calculated with total flux density. The only source that presents a remarkable difference between the core spectral in$\operatorname{dex}\left(\alpha_{\mathrm{r}}=0.14\right)$ and the total spectral index $\left(\alpha_{\mathrm{r}} \leq 0.72\right)$ is MS0922.9+7459.
Table 4. Total flux densities drawn from NVSS $(20 \mathrm{~cm})$ and EMSS $(6 \mathrm{~cm})$ archives for the 26 sources. NVSS peak flux density is also listed.

\begin{tabular}{l|rr|r}
\hline \hline & \multicolumn{2}{|c|}{ NVSS * } & EMSS * \\
& \multicolumn{2}{|c}{$(20 \mathrm{~cm})$} & $(6 \mathrm{~cm})$ \\
Name & $F_{\text {tot }}$ & $F_{\text {peak }}$ & $F_{\text {tot }}$ \\
\hline MS0122.1+0903 & 2.49 & 1.75 & 1.4 \\
MS0158.5+0019 & 12.7 & 12.7 & 11.3 \\
MS0205.7+3509 & 4.7 & 4.7 & 3.6 \\
MS0257.9+3429 & 10.1 & 10.1 & 10.0 \\
MS0317.0+1834 & 20.9 & 17.9 & 11.5 \\
MS0419.3+1943 & 2.85 & 2.76 & 8.0 \\
MS0607.9+7108 & 25.2 & 25.2 & 18.2 \\
MS0737.9+7441 & 22.7 & 22.7 & 24.0 \\
MS0922.9+7459 & 84.2 & 59.2 & 3.3 \\
MS0950.9+4929 & 2.6 & 2.6 & 3.3 \\
MS1019.0+5139 & 4.8 & 4.8 & 2.4 \\
MS1050.7+4946 & 64.1 & 64.1 & 53.8 \\
MS1207.9+3945 & 18.7 & 18.7 & 6.1 \\
MS1221.8+2452 & 24.5 & 24.5 & 26.4 \\
MS1229.2+6430 & 58.0 & 54.5 & 42.0 \\
MS1235.4+6315 & 12.1 & 12.1 & 7.0 \\
MS1402.3+0416 & 40.7 & 40.7 & 20.8 \\
MS1407.9+5954 & 35.7 & 33.3 & 16.5 \\
MS1443.5+6348 & 17.6 & 17.6 & 11.6 \\
MS1458.8+2249 & 31.9 & 31.9 & 29.8 \\
MS1534.2+0148 & 72.3 & 72.3 & 34.0 \\
MS1552.1+2020 & 79.0 & 79.0 & 37.5 \\
MS1757.7+7034 & 10.3 & 10.3 & 7.2 \\
MS2143.4+0704 & 101.1 & 95.1 & 50.0 \\
MS2336.5+0517 & 16.5 & 13.6 & 4.9 \\
MS2347.4+1924 & 4.7 & 4.7 & 3.2 \\
\hline$*$ Tota &
\end{tabular}

* Total and Peak Flux Density in mJy.

In Table 5, spectral indices are listed from nonsimultaneous and simultaneous spectra. The second column gives simultaneous slopes for the 26 sources of the CWSR sample; in the third, fourth and fifth column non-simultaneous slopes are computed, respectively, with $20 \mathrm{~cm} \mathrm{NVSS}$ and $6 \mathrm{~cm}$ EMSS observations, with $20 \mathrm{~cm}$ NVSS and $6 \mathrm{~cm} \mathrm{CWSR} \mathrm{ob-}$ servations, with $20 \mathrm{~cm}$ CWSR and $6 \mathrm{~cm}$ EMSS observations. By comparing different values of spectral slope, we note 
Table 5. Simultaneous and non-simultaneous spectral indices are listed for 26 objects $\left(S_{v} \propto v^{-\alpha_{\mathrm{r}}}\right)$.

\begin{tabular}{|c|c|c|c|c|}
\hline Name & $\begin{array}{r}\operatorname{sim} . \alpha_{\mathrm{r}} \\
\mathrm{CWSR} \\
- \\
\end{array}$ & $\begin{array}{l}\text { NVSS/ } \\
\text { EMSS }\end{array}$ & $\begin{array}{l}\text { Ion-sim. } \alpha_{\mathrm{r}} \\
\text { NVSS/ } \\
\text { CWSR }\end{array}$ & $\begin{array}{r}\text { CWSR/ } \\
\text { EMSS }\end{array}$ \\
\hline MS0122.1+0903 & 0.09 & 0.48 & 0.44 & 0.02 \\
\hline MS0158.5+0019 & 0.28 & 0.10 & 0.22 & 0.13 \\
\hline MS0205.7+3509 & -0.22 & 0.22 & -0.09 & -0.017 \\
\hline MS0257.9+3429 & -0.15 & 0.01 & 0.05 & -0.11 \\
\hline MS0317.0+1834 & 0.48 & 0.57 & 0.50 & 0.02 \\
\hline MS0419.3+1943 & -0.19 & -0.86 & -1.00 & -0.23 \\
\hline MS0607.9+7108 & 0.20 & 0.27 & 0.47 & -0.14 \\
\hline MS0737.9+7441 & 0.14 & -0.05 & 0.01 & 0.05 \\
\hline MS0922.9+7459 & 0.72 & 2.70 & 2.50 & 2.32 \\
\hline MS0950.9+4929 & 0.21 & -0.20 & -0.23 & 0.27 \\
\hline MS1019.0+5139 & -0.01 & 0.56 & 0.40 & 0.14 \\
\hline MS1050.7+4946 & 0.24 & 0.15 & 0.34 & 0.05 \\
\hline MS1207.9+3945 & 1.25 & 0.94 & 0.93 & 0.80 \\
\hline MS1221.8+2452 & 0.05 & -0.06 & 0.09 & -0.10 \\
\hline MS1229.2+6430 & 0.16 & 0.27 & 0.19 & 0.22 \\
\hline MS1235.4+6315 & 0.36 & 0.48 & 0.46 & 0.35 \\
\hline MS1402.3+0416 & 0.42 & 0.59 & 0.56 & 0.64 \\
\hline MS1407.9+5954 & 0.37 & 0.64 & 0.50 & 0.54 \\
\hline MS1443.5+6348 & 0.33 & 0.35 & 1.14 & -0.15 \\
\hline MS1458.8+2249 & -0.41 & 0.06 & -0.84 & 0.40 \\
\hline MS1534.2+0148 & 0.57 & 0.63 & 0.63 & 0.54 \\
\hline MS1552.1+2020 & 0.45 & 0.62 & 0.59 & 0.45 \\
\hline MS1757.7+7034 & 0.09 & 0.30 & -0.07 & 0.40 \\
\hline MS2143.4+0704 & 0.34 & 0.59 & 0.55 & 0.38 \\
\hline MS2336.5+0517 & 0.53 & 1.01 & 0.86 & 0.73 \\
\hline MS2347.4+1924 & 0.39 & 0.32 & 0.37 & 0.31 \\
\hline
\end{tabular}

that 11 of 26 objects have spectral indices that could be classified differently depending on the dataset used; in particularly, 9 of 11 sources could have a flat or steep spectrum, 1 source (MS0419+1943) could have an inverted or flat spectrum, 1 source (MS1458.8+2249) could have an inverted, flat or steep spectrum.

In the next section we will consider a few explanation that could affect these measurements: the well-known variability of these objects and the angular resolution of the observations. Data taken with different angular resolution in fact could measure, if present, a different amount of extended flux.

\section{Results}

\subsection{Core-to-total flux density ratio}

It is already known that BL Lacs are very core-dominated sources (e.g. Perlman \& Stocke 1993) and that this behavior is more evident in higher frequency observations. This is confirmed from our data (see Sect. 3.2.1). In fact, we confirm the trend of the core-dominance in the CWSR sample by fitting $F_{\text {peak }}$ vs. $F_{\text {tot }}$ (from Table 2) with the LSQ. The LSQ slope
Table 6. Results of the fit of $F_{\text {Peak }}$ vs. $F_{\text {Tot }}$ for the CWSR sources indicating that an extended component is visible at $20 \mathrm{~cm}$.

\begin{tabular}{lrr}
\hline \hline Frequency band & $\alpha$ & $\beta$ \\
\hline$L(20 \mathrm{~cm})$ & 0.94 & 0.02 \\
$L(20 \mathrm{~cm})^{*}$ & 0.96 & 0.01 \\
$C(6 \mathrm{~cm})$ & 0.99 & -0.03 \\
$X(3.6 \mathrm{~cm})$ & 1.00 & -0.06 \\
\hline * Fit without MS0922 $9+7459$ &
\end{tabular}

${ }^{*}$ Fit without MS0922.9+7459.

and intercept are given in Table 6: the first column gives the VLA frequency band, the second and the third column, respectively, give the slope and the intercept of the LSQ fit. We compute the fit also excluding the L band value of MS0922.9+7459 (that is confused, see Sect. 3.3)

The errors on the slopes are typically $0.1-0.2$, and therefore only at $20 \mathrm{~cm}$ there is a small deviation from a linear fit, indicating the presence of an extended component which is more visible for brighter sources.

We also checked for a possible redshift dependence of the core-dominance since extended surface brightness dims as $(1+z)^{-4}$, so significant amount of flux from the extended component could be lost at high $z$. In addition, since at large distances a given angular resolution corresponds to a larger physical size, if much of the extended flux of a source is close to the core, some of its inner part could be subsumed into the central component and therefore included with the measured core flux. Perlman \& Stocke (1993) studied the possible biases introduced by these terms and found that for sources with $z \leq 0.5$ there is only a modest correction. Since the objects in this work are all at $z \lesssim 0.5$, we estimate that the loss of the extended flux due to these biases was not significant and, thus, did not consider it.

\subsection{Spectral indices}

We studied the distribution of spectral slopes reported in Table 5. In Fig. 1 the four $\alpha_{\mathrm{r}}$ distributions obtained for the simultaneous and non-simultaneous datasets are shown. The dividing line at $\alpha_{\mathrm{r}}=0.5$ is plotted as a dashed line.

In Table 7 we report the percentage of sources with a spectrum steeper than $\alpha_{\mathrm{r}}=0.5$. The first column gives the different datasets used to compute the spectra; the second column gives the percentage of steep spectra computed; the third column gives the time span between the different datasets; the fourth column gives the VLA configurations used for the observations. As shown in Table 7, we found that about $15 \%$ of the sources have steep spectra computed with simultaneous data, and even larger percentages with non-simultaneous data. As shown in Fig. 1 the distribution of $\alpha_{\mathrm{r}}$ is a rather continuous one, so that picking a single value to divide it in two populations is rather arbitrary, and does not have a physical meaning. However, this has both practical and historical reasons (see e.g. the 1 Jy BL Lac sample definition in Kühr \& Schmidt 1990) and so it remains an important issue to be addressed. 
Table 7. The percentage of objects with steep spectra $\left(\alpha_{\mathrm{r}}>0.5 ; S_{v} \propto v^{-\alpha_{\mathrm{r}}}\right)$ is listed for different datasets.

\begin{tabular}{lcccc}
\hline \hline \multicolumn{2}{c}{ Datasets } & $\%$ & $\Delta$ & VLA \\
$20 \mathrm{~cm}$ & $6 \mathrm{~cm}$ & steep sp. & yrs & config. \\
\hline CWSR & CWSR & 15.4 & 0 & C+C/D \\
\hline NVSS & EMSS & 38.5 & $5-10$ & D, C \\
NVSS & CWSR & 38.5 & $4-7$ & D, C \\
CWSR & EMSS & 23.1 & $12-14$ & $\mathrm{C}+\mathrm{C} / \mathrm{D}, \mathrm{C}$ \\
\hline
\end{tabular}

${ }^{*}$ Also $3.6 \mathrm{~cm}$ observations in C configuration and 18 and $2 \mathrm{~cm}$ observations in $\mathrm{C} / \mathrm{D}$ configuration are used.
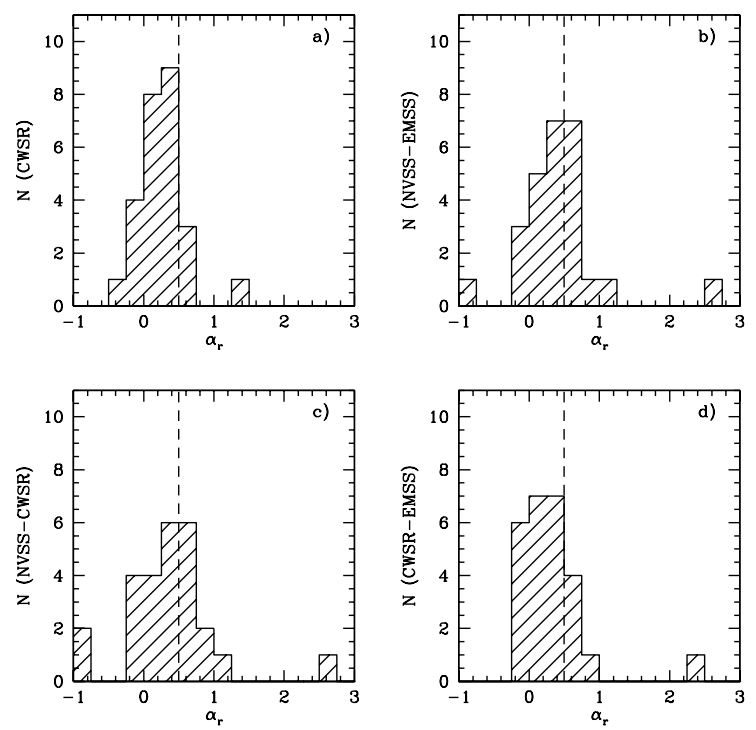

Fig. 1. The $\alpha_{\mathrm{r}}$ distributions for the 26 sources in the CWSR sample. Plot a), upper left, shows the spectral indices calculated from the simultaneous CWSR data. Plot b), upper right, shows the spectral indices calculated from $20 \mathrm{~cm}$ NVSS data and $6 \mathrm{~cm}$ EMSS data. Plot c), bottom left, shows the spectral indices calculated from $20 \mathrm{~cm}$ NVSS data and $6 \mathrm{~cm}$ CWSR data. Plot d), bottom right, shows the spectral indices calculated from $20 \mathrm{~cm}$ CWSR data and $6 \mathrm{~cm}$ EMSS data. The vertical dashed line represents the cut-off value $\alpha_{\mathrm{r}}=0.5$.

To investigate whether the different subsamples under consideration are consistent with each other, we performed a Kolmogorov-Smirnov test for the four $\alpha_{\mathrm{r}}$ distributions (see Fig. 1) by comparing their respective datasets considered in Table 7 . We cannot exclude that the four dataset combinations are extracted from the same population $(P>32 \%)$. However, the small number of points does not allow any further investigations.

Since by using non-simultaneous data we find a higher percentage of steep spectrum sources, it seems straightforward to ascribe this effect to flux variability, that we know is present in this class of objects. However, other factors are at play. Again from Table 7, we notice that the higher percentages of steep spectra correspond to the use of the $20 \mathrm{~cm}$ NVSS data, which were carried out with the VLA array in D configuration. As previously discussed (see Sect. 4.1), this probably indicates that a larger fraction of extended flux, which has a spectrum steeper than the core emission, is detected in these datasets.

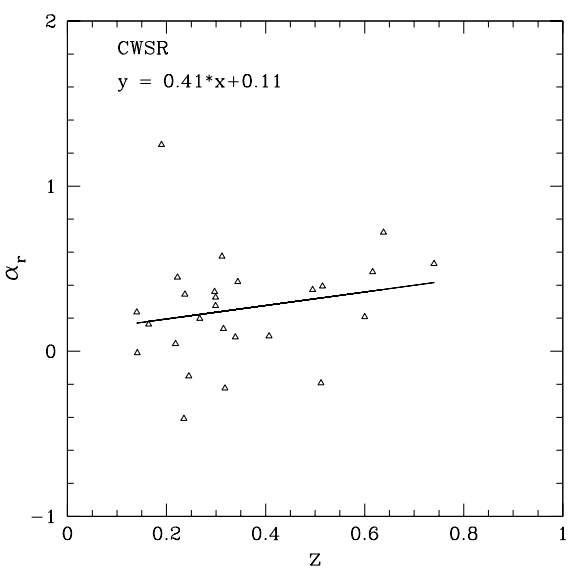

Fig. 2. $\alpha_{\mathrm{r}}$ against $z$ is plotted for the simultaneous dataset. The LSQ fit and the respective functional form are also shown.

The Gaussian distribution of the NVSS-to-CWSR $20 \mathrm{~cm}$ data ratio seems to confirm this hypothesis: the distribution mean value is $x_{\text {mean }}=1.19$, indicating that the NVSS flux densities exceed on average the CWSR values by $19 \%$.

The effect cannot be ascribed to confusion due to a large beam, since inspection of the higher resolution $\mathrm{C}$ configuration images shows that no sources fall into the NVSS beam beside the target BL Lac.

Thus, along with variability, we might suggest that a significant fraction of extended flux, that is considered negligible by BL Lacs beaming models (e.g. Blandford \& Rees 1978; Antonucci \& Ulvestad 1985), is present, and could at least partially explain the differences obtained in the $\alpha_{\mathrm{r}}$ distributions.

To investigate what influence the detection of an extended component has on the spectral index, we plotted the spectral slopes of the CWSR sources against redshift $z$ in Fig. 2. We confirm what we assumed in Sect. 4.1 that no redshift effect is present in the detection of extended flux due to decreasing of brightness. The LSQ fit in Fig. 2 is consistent with a constant due to the large error on slope $(0.41 \pm 0.39)$ and if anything sources at larger redshift seem to have steeper slopes.

To assess possible biases due to the varying spectral slope, we computed total luminosity for the 25 XBLs with known redshift in the $L, C$ and $X$ frequency bands. For the 22 CWSR sources observed on June 2000, peak and extended luminosities could also be computed from the peak and total flux densities; where extended emission was not detected, conservative upper limits on extended radio luminosity levels were 

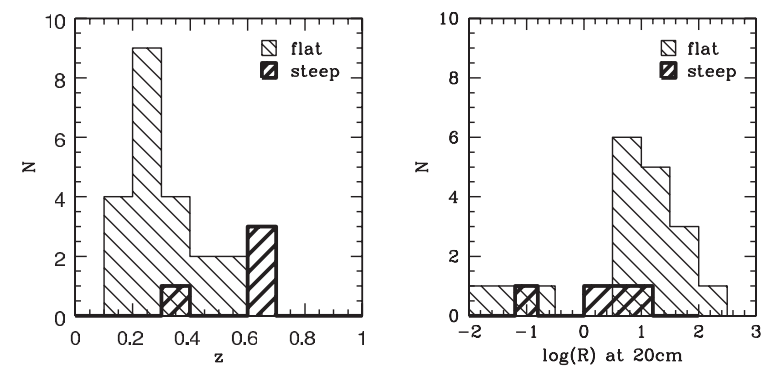

Fig. 3. The redshift left and core dominance right distribution of flat spectra objects compared to the steep spectra ones.

obtained by assuming that each source has uniformly bright extended emission at $1 \sigma$ detection level over a $3000 \mathrm{kpc}^{2}$ area surrounding the core (see Rector et al. 2000 for a description of how to compute the extended luminosities). The luminosity of the extended component is in general in agreement with what found previously (e.g. Rector et al. 2000; Perlman \& Stocke 1993) so we do not report values here. In fact the C configuration snapshot data do not have a sufficient $u v$ coverage to warrant a better definition of the core than the previous A configuration data on the same sources.

To test whether there is a correlation between luminosities and spectral slope, we computed the correlation coefficient for the LSQ fit and its goodness probability. We found again that there is a $99 \%$ probability that a correlation exists only for extended luminosity at $20 \mathrm{~cm}$. We check also a possible dependence of $\alpha_{\mathrm{r}}$ on NVSS and EMSS radio luminosities. The correlation between spectral slope and $20 \mathrm{~cm}$ luminosity seems to support the idea that the extended component is important in these objects and that this is the main factor to explain the difference in the spectral measures.

By splitting our sample of 26 objects in two groups, steep and flat spectra, we studied the different distribution of redshift, radio luminosity (at 20 and $6 \mathrm{~cm}), X$ luminosity $(0.3-3.5 \mathrm{keV}$ ) and $20 \mathrm{~cm}$ core dominance.

We plot in Fig. 3 (left) the histogram of the redshift distribution for objects with $\alpha_{\mathrm{r}}>0.5$ and $\alpha_{\mathrm{r}} \leq 0.5$ respectively. The flat-spectrum dataset has on average redshift values smaller than the steep-spectrum one. Since this is a flux limited sample, it is fair that we find the same behavior also for the radio and $\mathrm{X}$-ray luminosities. However we stress that the redshift range of this sample is small, and in particular lower than the radio selected samples like the $1 \mathrm{Jy}$.

In Fig. 3 (right), we show the histogram of core dominance for the two datasets, at $20 \mathrm{~cm}$. As expected, the steep spectrum sources show in general a smaller degree of core dominance, even if the statistical significance of the result is marginal.

We also plot in Fig. 4 the core-dominance at $20 \mathrm{~cm}$ against the radio spectral slope $\alpha_{\mathrm{r}}$, for the sources for which we have a measure (i.e. not an upper limit) for $R$. The two variables have a correlation coefficient of -0.63 , indicating that, within the available statistics, they are correlated at $>99 \%$.

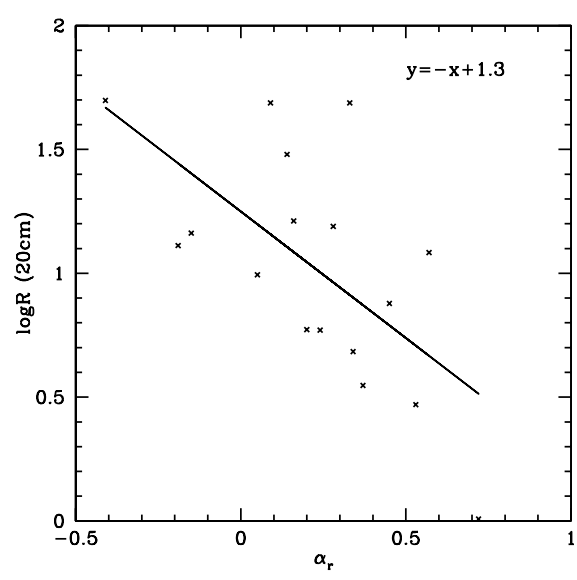

Fig. 4. The logarithm of core-dominance $R$ (at $20 \mathrm{~cm}$ ) is plotted against the radio spectrum $\alpha_{\mathrm{r}}$. The regression line has a slope of -1.02 with rather large uncertainties (0.32).

\section{3. $\left\langle V_{e} / V_{a}\right\rangle$ and the spectral index selection}

One controversial issue in the study of BL Lacs is their evolutionary behavior. The first studies were based on the EMSS and found evidence for "negative evolution" (i.e. less numerous/luminous objects in the past; Maccacaro et al. 1984; Morris et al. 1991; Wolter et al. 1994; Rector et al. 2000). At the same time the $1 \mathrm{Jy}$ sample (selected in the radio band, and with a constraint on $\alpha_{\mathrm{r}}<0.5$, Kühr \& Schmidt 1990) showed no or mild positive evolution (Stickel et al. 1991; Rector \& Stocke 2001). It is therefore interesting to think that the different criteria, in particular the flat radio spectral index, could select samples with different evolution properties. This result is obtained if the steep spectrum BL Lacs, present only in X-ray selected samples, all show a very negative evolution. Since the number involved in these samples are small (20-40 objects in total), even few objects have a large weight.

We used the standard $\left\langle V_{\mathrm{e}} / V_{\mathrm{a}}\right\rangle$ test (Avni \& Bachall 1980) to check the uniformity of the spatial distribution of the objects in a flux limited sample. For each object the two volumes $\left(V_{\mathrm{e}}\right.$ and $V_{\mathrm{a}}$ ) are derived on the basis of the observed redshift and the maximum redshift at which the object could be still included in the survey. A value of $\left\langle V_{\mathrm{e}} / V_{\mathrm{a}}\right\rangle=0.5$ is given by a uniform distribution, while lower values of $\left\langle V_{\mathrm{e}} / V_{\mathrm{a}}\right\rangle$ indicate a distribution skewed towards low redshifts. Details can be found e.g. in Morris et al. (1991). We stress here that only the X-ray flux limits are used to derive the maximum available volume. In fact the EMSS did not have any requirement on the radio flux or optical flux to have an object enter the sample, and all the EMSS BL Lacs have been also detected in the radio band.

To test the impact of the $\alpha_{\mathrm{r}}$ selection criteria on the measure of the evolution, we studied the correlation between $V_{\mathrm{e}} / V_{\mathrm{a}}$ and the radio spectral index $\alpha_{\mathrm{r}}$ for the 25 objects in the CWSR sample with a redshift measure (see Rector et al. 2000 for the $V_{\mathrm{e}} / V_{\mathrm{a}}$ values), plotted in Fig. 5. The data have been also grouped in three $\alpha_{\mathrm{r}}$ bins to better show the trend $\left(\alpha_{\mathrm{r}}<0.2\right.$; $\left.0.2<\alpha_{\mathrm{r}}<0.5 ; \alpha_{\mathrm{r}}>0.5\right)$ and plotted in Fig. 5 with error bars that reflect number statistics. We find that the mean value is consistent with a constant in the 3 intervals of $\alpha_{\mathrm{r}}$. If anything there is a slight suggestion that XBLs with steep spectra 


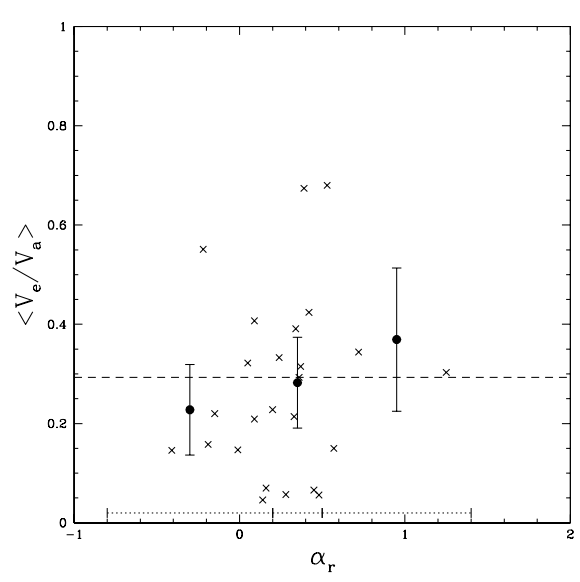

Fig. 5. $V_{\mathrm{e}} / V_{\mathrm{a}}$ against $\alpha_{\mathrm{r}}$ is shown for the $25 \mathrm{XBLs}$ with a redshift measure. The $\left\langle V_{\mathrm{e}} / V_{\mathrm{a}}\right\rangle$ trend is also plotted with error-bars for $\alpha_{\mathrm{r}}$ grouped in three bins $\left(\alpha_{\mathrm{r}}<0.2 ; 0.2<\alpha_{\mathrm{r}}<0.5 ; \alpha_{\mathrm{r}}>0.5\right)$. The dotted line represents the mean value of $\left\langle V_{\mathrm{e}} / V_{\mathrm{a}}\right\rangle$.

$\left(\alpha_{\mathrm{r}}>0.5\right)$ have a higher mean $\left\langle V_{\mathrm{e}} / V_{\mathrm{a}}\right\rangle$ value. Thus, it seems that the exclusion of steep spectra objects in RBLs samples could not cause the differences observed in the cosmological evolution with respect to the EMSS sample.

The picture is however far from clear: while surveys like the Deep X-ray Radio Blazar Survey (DXRBS; Perlman et al. 1998; Padovani et al. 2002; Giommi et al. 2002), the "sedentary" survey (Giommi et al. 1999) and the ROSAT - All Sky Survey (RASS; Bade et al. 1998; Beckmann et al. 2003) found very low values of $\left\langle V_{\mathrm{e}} / V_{\mathrm{a}}\right\rangle$ for XBLs, other surveys like the Radio Emitting X-ray survey (REX; Caccianiga et al. 1999, 2002) did not find the same result, possibly indicating that the negative evolution in this class of objects is not so strong as was previously measured.

\section{Conclusions}

By using fluxes at 20,6 and $3.6 \mathrm{~cm}$, simultaneously measured with the VLA, we computed the radio spectra for a complete subsample of 22 XBLs from the EMSS (Gioia et al. 1990; Stocke et al. 1991). To this sample we added 4 sources whose simultaneous spectra were obtained by Stocke et al. (1985). The aim was to study the spectral slope distribution at radio frequencies without possible biases introduced by the flat-radiospectrum criterion often used in selecting BL Lac samples. We found that about $15 \%$ of the sources have steep spectra. We considered the archived non-simultaneous data as well, finding an even higher percentage of steep spectra. This effect could be ascribed to variability, but other factors seem more significant in determining the measured slope of spectra. The higher percentage of steep spectra objects $(\sim 38 \%)$, obtained by using the $20 \mathrm{~cm}$ NVSS observations, implies the presence of a large fraction of extended flux that is preferentially detected by the VLA D configuration. This seems also supported by a possible correlation between spectral index and $20 \mathrm{~cm}$ extended luminosities, computed either with simultaneous or non-simultaneous data.

We find that the possible biases introduced by the flat-radiospectrum criterion in the RBLs samples cannot easily account for the discrepancies observed in the $\left\langle V_{\mathrm{e}} / V_{\mathrm{a}}\right\rangle$ values of RBLs and XBLs samples and therefore the issue of evolution of different BL Lac classes is still open. The percentages of steep spectrum BL Lacs found for the EMSS sample cannot be applied straightforwardly to RBL samples, since a) the selection by radio spectral index is sometimes computed using nonsimultaneous data; b) the XBLs properties are different from those of RBLs in the sense that RBLs are more variable and possibly more core dominated. If the observed spread in $\alpha_{\mathrm{r}}$ is mostly due to variability, we should expect a larger fraction of steep RBLs than XBLs. If most of the effect is due, as the data seem to suggest, to the presence of a significant extended component, that is lesser in RBL, the influence on the radio selected objects should be less severe.

We stress here that the $\alpha_{\mathrm{r}}$ distribution in the EMSS BL Lacs simultaneous data is a continuous one and so any division in two subsamples is somewhat arbitrary and does not have a physical underlying support. We are tempted to suggest a different cut-off value of $\alpha_{\mathrm{r}}=0.7$ (as done e.g. by Perlman et al. 1998 in DXRBS) that would then exclude $\leq 10 \%$ of all BL Lacs based upon our findings here. But, because this would also include a very large number of "normal" radio galaxies, some of which with optical featureless spectra (see e.g. discussions in Rector \& Stocke 2001; Perlman et al. 1996), other information would have to be used to define them as BL Lacs (e.g. variable optical polarization).

However, we have shown that the selection effects applied by using a cut-off value in the radio spectral index of $\alpha_{\mathrm{r}}=$ 0.5 are not so severe. The steeper BL Lacs that are excluded from this criterion seem to have the same overall properties of the flat ones, especially for what concerns the cosmological distribution. We can therefore conclude that the discrepancies found by using different samples cannot be ascribed to the flat radio spectrum criterion.

Acknowledgements. It is pleasure to thank Tommaso Maccacaro for stimulating discussions and suggestions. We thank the referee for his comments that helped improve the paper. This work has received partial financial support from the Italian Space Agency (ASI) and MIUR.

\section{References}

Angel, J. R. P., \& Stockman, H. S. 1980, ARA\&A, 18, 321 Antonucci, R. R. J., \& Ulvestad, J. S. 1985, ApJ, 294, 158

Avni, Y., \& Bahcall, J. N. 1980, ApJ, 235, 694

Baars, J. W. M., Genzel, R., Pauliny-Toth, I. I. K., \& Witzel, A. 1977, A\&A, 61, 99

Bade, N., Beckmann, V., Douglas, N. G., et al. 1998, A\&A, 334, 459

Beckmann, V., Engels, D., Bade, N., \& Wucknitz, O. 2003, A\&A, 401, 927

Blandford, R., \& Rees, M. J. 1978, in Pittsburgh Conference on BL Lac Objects, ed. A. M. Wolfe (Pittsburgh: University of Pittsburgh Press), 328

Caccianiga, A., Maccacaro, T., Wolter, A., Della Ceca, R., \& Gioia, I. M. 1999, ApJ, 513, 51

Caccianiga, A., Maccacaro, T., Wolter, A., Della Ceca, R., \& Gioia, I. M. 2002, ApJ, 566, 181

Condon, J. J., Cotton, W. D., Greisen, E. W., et al. 1998, AJ, 115, 1693 Fleming, T. A., Green, R. F., Jannuzi, B. T., et al. 1993, AJ, 106, 1729 Gioia, I. M., Maccacaro, T., Schild, R. E., et al. 1990, ApJS, 72, 567 Giommi, P., Menna, M. T., \& Padovani, P. 1999, MNRAS, 310, 465 
Giommi, P., Padovani, P., Perri, M., Landt, H., \& Perlman, E. 2002, Blazar Astrophysics with BeppoSAX and Other Observatories, ed. P. Giommi, E. Massaro, \& G. Palumbo, ESA-ESRIN, 133

Jannuzi, B. T., Smith, P. S., \& Elston, R. 1993, ApJS, 85, 265

Jannuzi, B. T., Smith, P. S., \& Elston, R. 1994, ApJ, 428, 130

Kühr, H., \& Schmidt, G. D. 1990, AJ, 99, 1

Landt, H., Padovani, P., Perlman, E. S., et al. 2001, MNRAS, 323, 757

Laurent-Muehleisen, S. A., Kollgaard, R. I., Moellenbrock, G. A., \& Feigelson, E. D. 1993, AJ, 106, 875

Maccacaro, T., Wolter, A., McLean, B., et al. 1994, ApL\&C, 29, 267

Marchã, M. J. M., Browne, I. W. A., Impey, C. D., \& Smith, P. S. 1996, MNRAS, 281, 425

Morris, S. L., Stocke, J. T., Gioia, I. M., et al. 1991, ApJ, 380, 49

Owen, F. N., Ledlow, M. J., \& Keel, W. C. 1996, AJ, 111, 53

Padovani, P., \& Giommi, P. 1995, ApJ, 444, 567

Padovani, P. 2002, Blazar Astrophysics with BeppoSAX and Other Observatories, ed. P. Giommi, E. Massaro, \& G. Palumbo, ESA-ESRIN, 101

Perley, R. A. 2000, The Very Large Array Observational Status Summary, ed. R. M. Hjellming
Perlman, E. S., \& Stocke, J. T. 1993, ApJ, 406, 430

Perlman, E. S., Stocke, J. T., Wang, Q. D., \& Morris, S. L. 1996, ApJ, 465,451

Perlman, E. S., Padovani, P., Giommi, P., et al. 1998, AJ, 115, 1253

Piccinotti, G., Mushotzky, R. F., Boldt, E. A., et al. 1982, ApJ, 253, 485

Rector, T. A., Stocke, J. T., \& Perlman, E. S. 1999, ApJ, 516, 145

Rector, T. A., Stocke, J. T., Perlman, E. S., Morris, S. L., \& Gioia, I. M. 2000, AJ, 120, 1626

Rector, T. A., \& Stocke, J. T. 2001, AJ, 122, 565

Stickel, M., Padovani, P., Urry, C. M., Fried, J. W., \& Kühr, H. 1991, ApJ, 374, 431

Stocke, J. T., Liebert, J., Schmidt, G., et al. 1985, ApJ, 298, 619

Stocke, J. T., Morris, S. L., Gioia, I. M., et al. 1991, ApJS, 76, 813

Ulrich, M. H., Maraschi, L., \& Urry, C. M. 1997, ARA\&A, 35, 445

Urry, C. M., \& Padovani, P. 1995, PASP, 107, 803

Watson, D., McBreen, B., Hanlon, L., et al. 2004, A\&A, 418, 459

Wolter, A., Caccianiga, A., Della Ceca, R., \& Maccacaro, T. 1994, ApJ, 433, 29 